\title{
Lessons learned in the IBL project
}

\author{
A. Miuccia ${ }^{\mathrm{a}}$, on behalf of the ATLAS Collaboration
}

${ }^{a}$ University of Geneva, Geneva, Switzerland

\begin{abstract}
The ATLAS experiment is ready for Run II of the LHC with improved tracking performance thanks to the installation of a new Pixel Detector layer, called the Insertable B-Layer (IBL). The IBL was installed in May 2014 located at only $3.3 \mathrm{~cm}$ radius from the beam axis and it has been successfully taking data since the beginning of Run II in June 2015. An overview of the lessons learned during the IBL construction will be presented, focusing on the challenges and highlighting the issues met during the production, integration, installation and commissioning phases of the detector.
\end{abstract}

Keywords: Tracking detectors, Silicon, Pixel detectors, LHC Run II PACS: 29.40.Wk, 29.40.Gx

\section{Introduction}

The Insertable B-layer (IBL) [1] [2] is a new layer of silicon pixel detectors that was inserted into ATLAS [3] during the first long shutdown of the LHC in addition to the ATLAS Pixel Detector [4]. The IBL has a cylindrical geometry, and it is made of 14 staves instrumented with silicon pixel modules which are located at an average distance of $3.3 \mathrm{~cm}$ from the beam axis. The IBL was added together with a new smaller radius beam-pipe. The main motivation of the IBL construction is to maintain the high performance of the Inner Detector tracking system despite expected inefficiencies due to high occupancy in the Pixel Detector B-Layer with increased luminosity in LHC Run II. In particular IBL together with the Pixel Detector B-layer ensures a higher tracking robustness, improving the impact parameter resolution and b-tagging efficiency. A new front end readout chip [5] was developed for the IBL, the FE-I4. Thanks to a revised electronic architecture with respect to the old chip, the FE-I4 is able to cope with the high occupancy scenario expected with increased LHC luminosities during Run II. Two technologies of silicon sensors are used in the IBL: a new version of the planar technology [6] with the inactive edges miNucl. Instrum. Meth.ized by shifting guard rings underneath the active pixel region and a novel 3D technology [7], which is used for the first time in a high energy particle physics experiment. The planar sensors were produced at $\mathrm{CiS}^{1}$ while the $3 \mathrm{D}$ sensors were produced at $\mathrm{FBK}^{2}$ and at $\mathrm{CNM}^{3}$. The IBL is successfully taking data in Run II of the LHC, since the beginning of June 2015.

${ }^{1} \mathrm{CiS}$ : Forschungsinstitut fur Mikrosensorik und Photovoltaik GmbH, Konrad-Zuse-Strasse 14, 99099 Erfurt, Germany. See http://www.cismst.org

${ }^{2}$ FBK: Fondazione Bruno Kessler (FBK), Via Sommarive 18, 38123 Povo di Trento, Italy. See http://www.fbk.eu

${ }^{3}$ CNM: Centro Nacional de Microelectronica (CNM-IMB-CSIC), Campus Universidad Autonoma de Barcelona, 08193 Bellaterra (Barcelona), Spain. See http://www.imbenm.csic.es

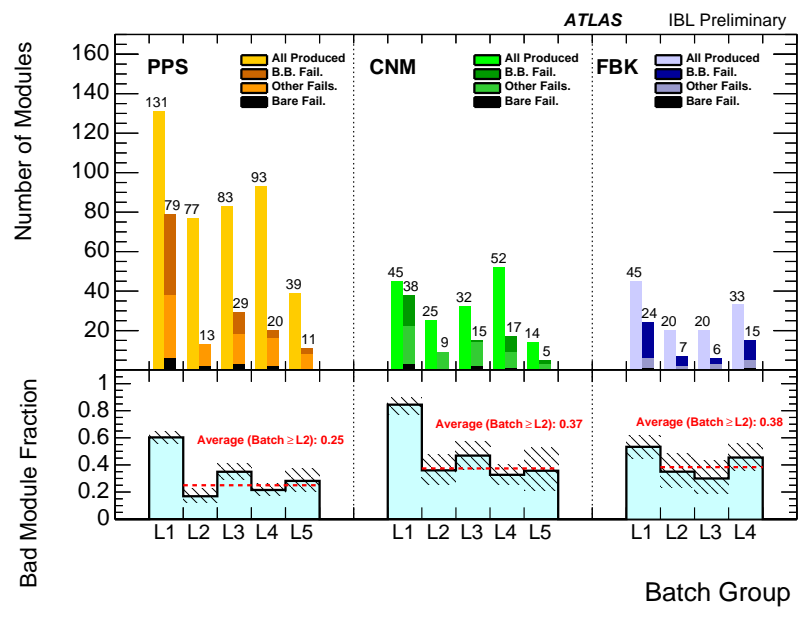

Figure 1: Yield of IBL module production for different sensor types. B.B. Fail. stands for bump-bonding failure, Bare Fail. stands for the mechanical accidents. PPS stands for Planar Pixel Sensors, while CNM and FBK are the two producers of $3 \mathrm{D}$ silicon sensors.

\section{Major lessons learned}

\subsection{Production yield of IBL modules}

Figure 1 shows the yield of IBL module assembly for the different sensor technologies. The main issues met during production were related to failures in the bump bonding process and to the selection of some of the 3D sensors from one of the vendors, which will be referred as 'A' in the following text. The first batch of IBL modules was largely affected by bump bonding defects, resulting in either open or merged bumps. While the investigation was underway, the production was temporarily stopped. A detailed analysis of the samples revealed polymerized flux residuals preventing a proper bump connection between sensor and FE pixel. The solder flux, used also as a tacking medium, was replaced with a glycerin compound which 
solved the problem. Another issue faced during the production was the rejection of a fraction of the 3D modules after the assembly. The 'A' sensor selection criteria were originally based on the leakage current measured through the guard ring structure that surrounds the pixel matrix. In this way the current measurement is sensitive to the edge of the sensor, but it is not indicative of the total leakage current. Initial studies indicated the same predictive power [8] between the breakdown voltage measured through the guard ring structure and the breakdown voltage after detector assembly, measured with the full pixel matrix. However, during module assembly, the correlation proved to be unreliable. All the remaining bare sensors were retested on a probe station, biasing the full matrix, and released to the flip chipping according to the leakage current specification. In this second test the entire pixel matrix was probed: the n-side of the sensor was placed in contact with the grounded chuck via the under-bump metallisation, while the p-side was connected to the bias potential.

\subsection{Wire-bonds oscillation}

The IBL, as part of the Inner Detector, is operating in a $2 T$ solenoid field, therefore the influence of the Lorentz force on the wire-bonds transporting DC and AC currents needed to be studied. The IBL wire bonds are exposed to a maximum force of $4 \times 10^{-4} N$ due to both DC component of the currents and the AC component coming from front end activity, which is smaller than the miNucl. Instrum. Meth.um force for breaking the wirebonds [9]. However, if this AC component has a frequency close to the wire resonance frequency, it will result in wire oscillation. Laboratory tests showed that for a maximum length of $2.8 \mathrm{~mm}$ an AC component of $100 \mathrm{~mA}$ amplitude generated oscillations at the frequency of about to $9.4 \mathrm{kHz}$, as shown in figure 2. Exposure to the resonance frequency for more than few minutes will break the wire. This issue was discovered late in the production; the possibility to protect the wires by potting $^{4}$ was considered, but discarded because risky. In order to avoid any potentially dangerous frequency for the wire-bonds a Fixed Frequency Trigger Veto (FFTV) has been implemented in the readout chain. The FFTV prevents the front end electronics from being triggered by a frequency in the range of frequency from 2 to $40 \mathrm{kHz}$, for more than 10 consecutive times.

\subsection{Wire-bonds corrosion}

During the IBL production the presence of crystalline residues was discovered on the flex of the stave next to the wirebond feet. This was identified to be a corrosion process [10] of the wire-bonds which was traced to condensation during one of the stave quality assurance steps. Further analysis has shown the presence of halogens on the flex and galvanic coupling between the aluminum wire bond and the gold pad of the flex. This phenomenon could be reproduced in the laboratory in the presence of deionized water on wire bonded samples. This problem occurred during the thermal cycling steps in a climate

\footnotetext{
${ }^{4}$ Potting is meant as an encapsulation of sensitive components as wire-bonds in a resin for protection
}

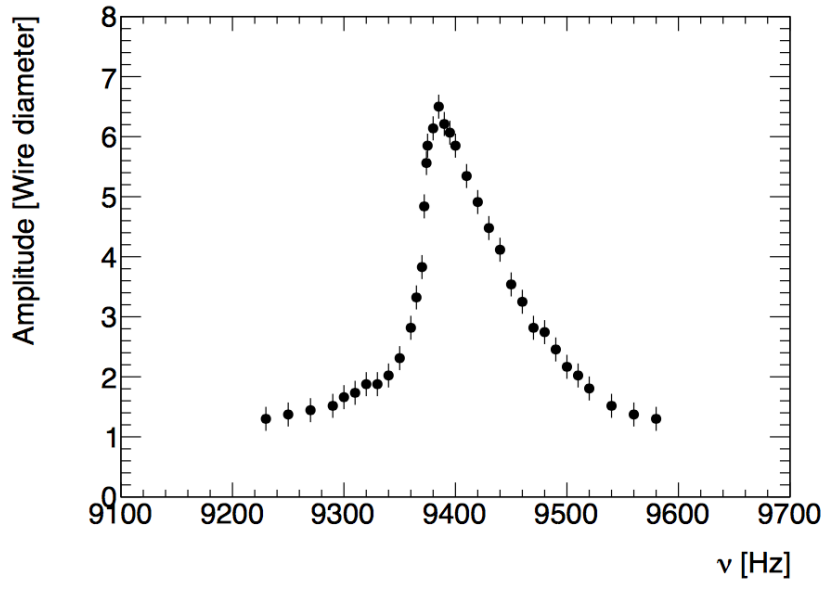

Figure 2: Oscillation amplitude expressed in wire diameter $(25 \mu \mathrm{m})$ as a function of the frequency for a wire of $2.8 \mathrm{~mm}$ length. The picture is taken from the Cern Technical note reported in [9]

chamber, which is an important part of the mechanical integrity checks. The problem was related to the temperature ramps in which a small exposure to humidity was found. Even if the volume was flushed with dry air during the cycles and the humidity control activated, this was not enough to guarantee the absence of water. As a consequence of this investigation all the newly loaded staves were not thermally cycled and all the staves which were affected by the condensation were cleaned and totally rebonded; the wire-bond quality was proven by a pull test campaign for the entire IBL stave production. The tests performed during the production demonstrated that the corrosion will happen only in case of condensation; the IBL detector is permanently flushed with nitrogen in the ATLAS volume and all the temperatures and humidities are constantly monitored. While the coldest temperature expected for the IBL is $\sim-35^{\circ} C$, the dew point measured at the exhaust of the IBL volume is always monitored and below $-70^{\circ} \mathrm{C}$.

\section{Conclusions}

The ATLAS experiment is now equipped with a fourth pixel layer which will provide redundancy to the B-layer measurements and which will improve tracking performance. Several challenges were met during the production chain and dedicated task forces were put in place to investigate and to overcome the issues. These experiences and the documentation [11], still in publication process at the date of this paper, of the various problems will be essential inputs for the construction of new future silicon trackers. The IBL detector is taking part in ATLAS Run II with excellent performance since the first $13 \mathrm{TeV}$ stable beam collisions, which happened in June 2015.

\section{References}

[1] The ATLAS Collaboration, ATLAS Insertable B-Layer Technical Design Report, CERN-LHCC-2010-013 
[2] The ATLAS Collaboration, ATLAS Insertable B-Layer Technical Design Report Addendum, CERN-LHCC-2012-009

[3] The ATLAS collaboration, The ATLAS experiment at the CERN Large Hadron Collider, JINST 3 (2008) S08003

[4] G. Aad et al., ATLAS pixel detector electronics and sensors, JINST 3 (2008) P07007

[5] M. Garcia-Sciveres et al., The FE-I4 pixel readout integrated circuit, Nucl. Instrum. Meth. A, 636 (2009), p. S155

[6] C. Goessling et al., Planar n+-in-n silicon pixel sensors for the ATLAS IBL upgrade, Nucl. Instrum. Meth. A, 650 (2011), p. 198

[7] C. Da Vià et al., 3D silicon sensors: Design, large area production and quality assurance for the ATLAS IBL pixel detector upgrade, Nucl. Instrum. Meth. A, 694 (2012), p. 321.

[8] The ATLAS IBL Collaboration, Prototype ATLAS IBL Modules using the FE-I4A Front-End Readout Chip, 2012 JINST 7 P11010

[9] D. Alvarez Feito et al. Studies of IBL wire bonds operation in a ATLASlike magnetic field, CERN-PH-EP-Tech-Note-2015-002

[10] Private communication with Rui De Oliveira, Alan Honma and with support of CERN PH-DT

[11] The ATLAS Collaboration, Production and Integration of the ATLAS Insertable B-Layer, in preparation 